\title{
Adoption and diffusion of cost reducing innovations: Cournot competition in duopoly*
}

\author{
Raouf Boucekkine $^{\dagger} \quad$ Omar Licandro ${ }^{\ddagger} \quad$ Antonio Minniti ${ }^{\S}$ \\ October 5, 2004
}

\begin{abstract}
This note analyses the adoption and diffusion of innovations in a horizontally differentiated Cournot duopoly in which firms have to choose the dates for adopting a cost-reducing new technology like in Reinganum (1981a). We prove that product differentiation crucially matters in the diffusion pattern of the innovation and in the comparison between the adoption timing in the decentralized economy Vs the social optimum.
\end{abstract}

Keywords: Adoption, diffusion, differentiated duopoly

JEL-Classification: 031

*Boucekkine and Minniti acknowledge the financial support of the Belgian research programmes "Poles d'Attraction inter-universitaires" PAI P5/21, and "Action de Recherches Concertée" 03/08-302. Licandro acknowledges the financial support of DGCYT, project SEC2000-0260.

${ }^{\dagger}$ IRES and CORE, Université catholique de Louvain. boucekkine@ires.ucl.ac.be

$\ddagger$ Corresponding author. European University Institute, Villa San Paolo, Via della Piazzuola, 43, I - 50133 Firenze (Italy). omar.licandro@iue.it

${ }^{\S}$ CORE and IRES, Université catholique de Louvain. minniti@core.ucl.ac.be 


\section{Introduction}

Technology adoption is nowadays considered as a key factor explaining differences in productivity at all levels. Just like the capacity to innovate, the ability of industries to adopt new and more efficient technologies is a decisive determinant of their viability. A salient feature of the adoption patterns is the so-called technological diffusion, namely the sequential adoption of innovations by firms. This feature has been intensively studied in all the fields of economic theory, and several arguments have been put forward to explain the sequentiality of adoption. Among them, the strategic arguments have become increasingly popular. Reinganum (1981a, 1981b) are the seminal papers in this area. In the first paper, the author develops a game-theoretic approach to diffusion: In a duopoly where each firm must determine when to adopt (if ever) a cost-reducing innovation, she shows that even in the case of identical firms and complete certainty, two asymmetric Nash equilibria arise in pure strategy, one firm adopting relatively earlier, the other relatively later, that is diffusion occurs. In the second paper, Reinganum extends her results to the oligopoly case, which allows her to characterize explicitly the effects of market structure (as captured by the number of firms) on the diffusion patterns. In a subsequent development based on Reinganum (1981a, 1981b), Quirmbach (1986) compares diffusion rates for different market structures in the capital equipment market.

In this note, we use the framework of Reinganum (1981a) to analyse the impact of product differentiation on the timing of adoption. Our analysis is based on a horizontally differentiated duopoly with Cournot competition. There is an obvious motivation behind the extension of Reinganum's strategic set-up: The degree of differentiation of the produced goods is inversely related to competition toughness, and the latter is likely to play a central role in the adoption sequence since it influences a firm's pre-adoption profits as well as its post adoption profits. Therefore, our framework is a natural one to study the interaction between competition in the product market and technological diffusion.

We shall show that the degree of product differentiation has some crucial consequences on the adoption patterns either in the decentralized equilibrium or in the social planner problem's outcomes. The most striking results are twofold. First, we identify an inversely $U-$ shaped relationship between the first adoption and competition toughness in the product market, which is in sharp contrast for example to Götz (1999) who shows that increased com- 
petition often fastens diffusion in a market with monopolistic competition. Second, we show that while the first adoption is always delayed in the decentralized economy compared to the second best, full adoption (i.e. adoption by both firms) crucially depends on the degree of product differentiation. In particular, we prove that full adoption occurs earlier in the second best if the products are differentiated enough.

The paper is organized as follows. Section 2 gives the basic set-up. Section 3 derives the adoption decisions in the decentralized Vs second-best equilibria. Section 4 and 5 compare the outcomes of both equilibria in terms of technological diffusion and connect them with the underlying competition toughness in the product market.

\section{The basic set up}

The demand side is a simplified version of Singh and Vives (1984). Firm $i$, for $i=\{1,2\}$, produces a differentiated product, $q_{i}$, and sells it at price $p_{i}$. The demand functions for the differentiated products are assumed to be generated by a representative consumer with a quadratic utility function $U\left(q_{1}, q_{2}\right)=\alpha\left(q_{1}+q_{2}\right)-\frac{1}{2}\left(q_{1}^{2}+2 \gamma q_{1} q_{2}+q_{2}^{2}\right)$, with $\alpha>0$ and $\gamma \in(0,1]$. The parameter $\gamma$ indicates the strength of product differentiation: in the limit case $\gamma=1$, both products are perfect substitutes; instead, as $\gamma$ approaches zero, products become more differentiated. Maximizing consumers' utility yields the linear inverse demand schedule:

$$
p_{i}=\alpha-q_{i}-\gamma q_{j}
$$

with $i, j=\{1,2\}$ and $i \neq j$.

The two firms start with the same constant marginal cost $c<\alpha$. At time $t=0$, a new technology becomes available and its adoption reduces marginal costs to $c-\varepsilon$, with $\varepsilon \in(0, c)$. Firms decide on whether and when to adopt the new technology. At the status quo, (pre-innovation) instantaneous profits are denoted by $\pi>0$. When the innovation is adopted, adopter's profits (excluding adoption costs) become $\pi_{a}(s)$, depending on the number of adopters $s=\{1,2\}$, and non adopter's profits become $\pi_{n}(s)$, with $s=\{0,1\}$. Naturally, $\pi=\pi_{n}(0)$. In the following, let us assume that innovations are

non drastic, i.e. $\varepsilon \in\left(0, \frac{2-\gamma}{\gamma}(\alpha-c)\right)$; as shown below, under non drastic 
innovations non adopters are always optimally operative. ${ }^{1}$

In Cournot competition, firms choose output in order to maximize their respective market profits and the Cournot-Nash equilibrium is:

$$
q_{i}^{*}=\frac{2\left(\alpha-c_{i}\right)-\gamma\left(\alpha-c_{j}\right)}{\left(4-\gamma^{2}\right)}
$$

implying that:

$$
\begin{array}{|l|l|}
\hline \hline \pi=\pi_{n}(0)=\frac{(\alpha-c)^{2}}{(2+\gamma)^{2}} & \pi_{a}(2)=\frac{(\alpha-c+\varepsilon)^{2}}{(2+\gamma)^{2}} \\
\pi_{a}(1)=\frac{[(\alpha-c)(2-\gamma)+2 \varepsilon]^{2}}{\left(4-\gamma^{2}\right)^{2}} & \pi_{n}(1)=\frac{[(\alpha-c)(2-\gamma)-\gamma \varepsilon]^{2}}{\left(4-\gamma^{2}\right)^{2}} \\
\hline \hline
\end{array}
$$

Observe that adoption by one firm has a negative impact on the profits of the other firm (business stealing effect). Now, to simplify the exposition of the main results, we introduce the following notation:

i) $\Delta_{1}=\pi_{a}(1)-\pi$ representing the initial gain of the first adopter;

ii) $\Delta_{2}=\pi_{a}(2)-\pi_{n}(1)$ representing the gain of the second adopter;

iii) $\Delta_{3}=\pi-\pi_{n}(1)$ representing the profit decrease of the second adopter after the first adoption;

iv) $\Delta_{4}=\pi_{a}(1)-\pi_{a}(2)$ representing the profit decrease of the first adopter after the second adoption.

Demand and cost specifications in this paper satisfy Assumptions 1-3 in Reinganum (1981a). Consequently, firms have incentives to preemptive adoption, since the gain induced by the first adoption is larger than the gain induced by the second adoption, or:

$$
\Delta_{1}>\Delta_{2}
$$

\footnotetext{
${ }^{1}$ If the innovation were drastic, i.e. if $\varepsilon>\frac{2-\gamma}{\gamma}(\alpha-c)$, the reaction functions of the adopter and the non adopter never cross, implying that the non adopter optimally shuts down at the time of adoption, and the adopter becomes a monopolist. The main results of this paper do not change under drastic innovations.
} 
As outlined by Quirmbach (1986, Proposition 1, p. 35), this represents a necessary condition for diffusion. By rearranging terms in $\Delta_{1}$ and $\Delta_{2}$, Property 1 can be also written as:

$$
\Delta_{4}>\Delta_{3}
$$

i.e. the loss in terms of profits of the first adopter after the second adoption is larger than the one induced by the first adoption on the profits of the second adopter.

\section{Adoption}

The setting of the adoption game is close to Reinganum (1981a). Let $X(T)$ denote the undiscounted cost of adoption, i.e. the cost of bringing the new technology on line by date $T$. It is assumed that $X^{\prime}(T)<0$ and $X^{\prime \prime}(T)>0$, i.e. adoption costs decrease with the time elapsed until adoption, but at a declining rate. Moreover, $X(0)$ is assumed to be sufficiently large so that an initial adoption appears unattractive and $\lim _{t \rightarrow \infty} X(T)=0$, implying that adoption will eventually occur. At date $T=0$, firms must simultaneously precommit to an adoption date. ${ }^{2}$

In the next two subsections, we derive the noncooperative and the second best solutions of the model.

\subsection{The noncooperative equilibrium of the model}

Firm $i$ maximizes the discounted value of total profits $\Pi_{i}\left(T_{i}, T_{j}\right)$ by choosing the adoption date $T_{i}$. The payoff of duopolist $1, \Pi_{1}\left(T_{1}, T_{2}\right)$, is equal to:

$$
\begin{aligned}
& \int_{0}^{T_{1}} \pi e^{-r t} \mathrm{~d} t+\int_{T_{1}}^{T_{2}} \pi_{a}(1) e^{-r t} \mathrm{~d} t+\int_{T_{2}}^{\infty} \pi_{a}(2) e^{-r t} \mathrm{~d} t-X\left(T_{1}\right) e^{-r T_{1}} \text { if } T_{1} \leqq T_{2}, \\
& \int_{0}^{T_{2}} \pi e^{-r t} \mathrm{~d} t+\int_{T_{2}}^{T_{1}} \pi_{n}(1) e^{-r t} \mathrm{~d} t+\int_{T_{1}}^{\infty} \pi_{a}(2) e^{-r t} \mathrm{~d} t-X\left(T_{2}\right) e^{-r T_{2}} \text { if } T_{2} \leqq T_{1},
\end{aligned}
$$

\footnotetext{
${ }^{2}$ This corresponds to assume infinitely long information lags or, technically, an openloop information structure. In other words, a firm is not allowed to react to its rival's action during the game. The case without precommitment was introduced to the literature by Fudenberg and Tirole (1985). They show that a first-mover advantage will stimulate preemptive adoption until firms' payoffs are equalized if firms are unable to precommit to future actions (closed - loop information structure).
} 
where $r$ is the market interest rate. The payoff of firm 2 is:

$$
\Pi_{2}\left(T_{1}, T_{2}\right)=\Pi_{1}\left(T_{2}, T_{1}\right) .
$$

As shown by Reinganum (1981a, Assumption 5a), if $\Delta_{2}<-X^{\prime}(0)$, this duopoly game presents two Nash equilibria in pure strategies, with $0 \leq \hat{T}_{1}<$ $\hat{T}_{2}$ or $0 \leq \hat{T}_{2}<\hat{T}_{1}$, where $\hat{T}_{i}$ refers to firm $i$ 's optimal choice. This has two implications. Firstly, the diffusion of technologies is never instantaneous. Secondly, regardless of the fact that firms are identical, they never adopt the new technology at the same date. Observe that the analysis is restricted to the case of gross substitutability, i.e. $\gamma \in(0,1]$. In the case of gross complementarity, i.e. $\gamma \in(-1,0)$, Properties 1 and 2 are violated implying simultaneous adoption.

Since firms are identical, without loss of generality, we can assume that firm 1 adopts first; this means that $\hat{T}_{1}<\hat{T}_{2}$. The optimization problems are the following:

$$
\begin{aligned}
& \hat{T}_{1}=\arg \max _{T_{1}} \Pi_{1}\left(T_{1}, T_{2}\right), \\
& \hat{T}_{2}=\arg \max _{T_{2}} \Pi_{2}\left(T_{1}, T_{2}\right) .
\end{aligned}
$$

In other words, the objective of each firm is to choose an adoption date so as to maximize its discounted payoff, taking the choice of the other firm as given. Considering interior solutions, the first order conditions of these maximization problems are: ${ }^{3}$

$$
\begin{aligned}
& \Delta_{1}=-X^{\prime}\left(\hat{T}_{1}\right), \\
& \Delta_{2}=-X^{\prime}\left(\hat{T}_{2}\right) .
\end{aligned}
$$

Equations (1) and (2) state that the optimal adoption date equalizes the firm's benefits from adoption to the the marginal cost of waiting. Given that function $X^{\prime}\left(\hat{T}_{i}\right)$ is increasing in $\hat{T}_{i}$ and from Property $1, \hat{T}_{1}<\hat{T}_{2}$. Since $\Delta_{1}$ is a function of $\gamma$, we can study the way in which product differentiation influences the incentives and the timing of the first adoption. We state the following proposition:

\footnotetext{
${ }^{3}$ The second order conditions are satisfied since firm $i$ 's objective function is concave in variable $T_{i}$.
} 
Proposition 1 The relation between the adoption date $\hat{T}_{1}$ and the degree of product differentiation is $U$ - shaped.

Proof. Eq. (1) implies that $\frac{d \hat{T}_{1}}{d \gamma}=\frac{\frac{\partial \Delta_{1}}{\partial \gamma}}{-X^{\prime \prime}\left(\hat{T}_{1}\right)}$. Since the denominator of the previous ratio is negative, $\frac{d \hat{T}_{1}}{d \gamma}$ is inversely related to $\frac{\partial \Delta_{1}}{\partial \gamma}$. Now, differentiating $\Delta_{1}$ with respect to $\gamma$ gives us:

$$
\frac{\partial \Delta_{1}}{\partial \gamma} \lesseqgtr 0 \text { if and only if } \gamma \lesseqgtr \bar{\gamma}
$$

where $\bar{\gamma}=\frac{2\left[2(\alpha-c)+\varepsilon-\sqrt{(\alpha-c)^{2}+4 \varepsilon(\alpha-c)+\varepsilon^{2}}\right]}{3(\alpha-c)} \in(0,1)$

Since firms receive maximal (monopoly) profits when they produce independent products and obtain minimal ones if the good is homogeneous, the degree of product differentiation can be interpreted as inversely related to the degree of competition. Therefore, according to Proposition 1, competition toughness stimulates diffusion when products are substitute enough. Note that Götz (1999, pp. 689-691) obtains that increased competition promotes diffusion by means of a numerical simulation in a model of technological adoption with monopolistic competition. Instead, Proposition 1 suggests that in a differentiated duopoly the relation between the optimal timing of the first adopter and competition toughness is inversely $U-$ shaped. The reason of this non-monotonicity relies on the fact that product differentiation influences the incentive to adopt, $\Delta_{1}$, by changing both pre-innovation profits $\pi$ and post-innovation profits $\pi_{a}(1)$; in fact, an increase in $\gamma$ exerts a negative effect on $\pi$ and two opposite effects on $\pi_{a}(1)$. More precisely, the adverse effect on $\pi$ and $\pi_{a}(1)$ is due to the fact that a reduction in the degree of product differentiation decreases profits (usual competition effect); however, there is an additional (positive) effect on $\pi_{a}(1)$ since the first adopter is able to exploit more its cost advantage by discouraging the rival's production when competition is tougher. The obtained non-monotonic relationship is the result of these three effects. When $\gamma$ is lower than $\bar{\gamma}$, an increase in $\gamma$ reduces $\pi_{a}(1)$ more than $\pi$ meaning that $\Delta_{1}$ becomes smaller; the converse happens when $\gamma$ is higher than $\bar{\gamma}$.

We shall discuss the effects of product differentiation on the full adoption process in Sections 4 and 5. 


\subsection{The welfare analysis}

Now, we turn to welfare analysis in order to compute adoption timings in the second best framework. Because we focus on the optimal diffusion pattern, we assume that the social planner is unable to influence the pricing behaviour of the firms. The total surplus is written as:

$$
\begin{aligned}
W\left(T_{1}, T_{2}\right)= & \int_{0}^{T_{1}}[2 \pi+C] e^{-r t} \mathrm{~d} t+\int_{T_{1}}^{T_{2}}\left[\pi_{a}(1)+\pi_{n}(1)+C(1)\right] e^{-r t} \mathrm{~d} t+ \\
& +\int_{T_{2}}^{\infty}\left[2 \pi_{a}(2)+C(2)\right] e^{-r t} \mathrm{~d} t-X\left(T_{1}\right) e^{-r T_{1}}-X\left(T_{2}\right) e^{-r T_{2}}
\end{aligned}
$$

where

$$
\begin{gathered}
C=(1+\gamma) \pi, \\
C(1)=\pi_{a}(1) / 2+\pi_{n}(1) / 2+\gamma \sqrt{\pi_{a}(1) \cdot \pi_{n}(1)}, \\
C(2)=(1+\gamma) \pi_{a}(2),
\end{gathered}
$$

represent the consumer surplus ${ }^{4}$ when the number of adopters is zero, one and two, respectively.

The second best adoption dates result from maximizing $W\left(T_{1}, T_{2}\right)$ with respect to $T_{1}$ and $T_{2}$; the first order conditions are:

$$
\begin{gathered}
\Delta_{1}-\Delta_{3}+C(1)-C=-X^{\prime}\left(\tilde{T}_{1}\right), \\
\Delta_{2}-\Delta_{4}+C(2)-C(1)=-X^{\prime}\left(\tilde{T}_{2}\right) .
\end{gathered}
$$

Equation (3) states that the optimal choice of $\tilde{T}_{1}$ equalizes the social benefit ${ }^{5}$ of the first adoption to the marginal cost of postponing. A similar interpretation can be given to equation (4) for the second adoption date $\tilde{T}_{2}$.

\section{Timing of adoption in decentralized Vs sec- ond best equilibria}

In this section we turn to the question of how the timing of adoption in a decentralized economy differs from the second best. A similar issue has been

\footnotetext{
${ }^{4}$ Consumer surplus is equal to $U\left(q_{1}, q_{2}\right)-p_{1} q_{1}-p_{2} q_{2}=q_{1}^{2} / 2+q_{2}^{2} / 2+\gamma q_{1} q_{2}$.

${ }^{5}$ For instance, in a social welfare analysis, the benefit of the first adoption is equal to the net gain of being first, $\Delta_{1}-\Delta_{3}$ (look at Property 1), and the change in consumer surplus, $C(1)-C$.
} 
tackled by Quirmbach (1986) in the case of homogenous products. In the noncooperative equilibrium, adoption decisions are taken by firms. For this reason, optimal conditions (1) and (2) only consider gains of the adopters, $\Delta_{1}$ and $\Delta_{2}$. In contrast, the second best rules, also take into account the gains and losses of the other market agents, i.e., the other firm and consumers. Losses of the non adopter at the time of the first adoption are given by $\Delta_{3}$ and losses of the first adopter at the time of the second adoption are given by $\Delta_{4}$. Consumers' gains are given by the differences $C(1)-C>0$ at the time of the first adoption and $C(2)-C(1)>0$ at the time of the second adoption. This implies that for comparing the decentralized solution to the second best solution, we can simply look at the sign of $\left[C(1)-C-\Delta_{3}\right]$ and $\left[C(2)-C(1)-\Delta_{4}\right]$. The comparisons between equations (1) and (3), and between equations (2) and (4) give the following results:

Proposition 2 i) In the market economy, the new technology is introduced by the first adopter later than in the second best; that is:

$$
\hat{T}_{1}>\tilde{T}_{1} \text { for all } \gamma \in(0,1] .
$$

ii) Full adoption (by firm 2) happens earlier in the second best than in the decentralized economy if the products are sufficiently differentiated. More precisely:

$$
\hat{T}_{2}>\tilde{T}_{2} \text { if and only if } \gamma<\gamma^{*}=\frac{\alpha-c+\varepsilon / 2}{\alpha-c+\varepsilon} .
$$

Proof. We are interested in determining the sign of $\left[C(1)-C-\Delta_{3}\right]$ and $\left[C(2)-C(1)-\Delta_{4}\right]$; at this regard, we get that:

$$
\begin{gathered}
{\left[C(1)-C-\Delta_{3}\right]=\frac{\varepsilon[2(1-\gamma)(\alpha-c)+\varepsilon]}{2\left(4-\gamma^{2}\right)},} \\
{\left[C(2)-C(1)-\Delta_{4}\right]=\frac{\varepsilon[2(1-\gamma)(\alpha-c)+(1-2 \gamma) \varepsilon]}{2\left(4-\gamma^{2}\right)} .}
\end{gathered}
$$

Observe that $\left[C(1)-C-\Delta_{3}\right]>0$ for all $\gamma \in(0,1]$; this means that, for the first adoption, there are larger incentives to adopt in the second best than the decentralized economy. Instead, the sign of $\left[C(2)-C(1)-\Delta_{4}\right]$ is ambiguous; it can be positive or negative depending on the values of $\gamma$. Now, if both products are almost independent, i.e. $\gamma=\delta$, where $\delta>0$ but very 
small, then $\Delta_{3}$ and $\Delta_{4}$ are close to 0 ; in fact, each firm behaves as a monopolist and its profits do not depend on the adoption decisions of the other firm. Since the consumers always profit from any improvement in technology, $C(2)-C(1)$ is strictly positive; by continuity, when $\gamma$ is close to 0 , we have $\left[C(2)-C(1)-\Delta_{4}\right]>0$. Moreover, $\partial\left[C(2)-C(1)-\Delta_{4}\right] / \partial \gamma<0$ for all $\gamma \in(0,1]$; that is, $\left[C(2)-C(1)-\Delta_{4}\right]$ decreases in $\gamma$. To complete the proof we need to show that $\left[C(2)-C(1)-\Delta_{4}\right]<0$ when $\gamma$ is sufficiently large. This is simple to check; for instance, consider the other extreme situation of perfect substitution, i.e. $\gamma=1$. In such a case, $\left[C(2)-C(1)-\Delta_{4}\right]$ equals $-\varepsilon^{2} / 6$. We conclude that there exists a critical value of $\gamma$, denoted as $\gamma^{*}$, such that the term $\left[C(2)-C(1)-\Delta_{4}\right]$ is positive (negative) when $\gamma$ is lower (higher) than $\gamma^{*}$.

Therefore, whatever $\gamma$ is, the first adoption is always delayed in the decentralized economy. This is not always true for the full adoption since in this case the degree of product differentiation matters.

\section{The diffusion delay}

In this section we focus on the diffusion delay that is the time elapsed between the two dates of adoption. At this regard, in the noncooperative equilibrium consider the following function:

$$
Z\left(\hat{T}_{1}, \hat{T}_{2}\right) \equiv \frac{X^{\prime}\left(\hat{T}_{1}\right)}{X^{\prime}\left(\hat{T}_{2}\right)}=\frac{\Delta_{1}}{\Delta_{2}}
$$

with $\partial Z\left(\hat{T}_{1}, \hat{T}_{2}\right) / \partial \hat{T}_{1}<0$ and $\partial Z\left(\hat{T}_{1}, \hat{T}_{2}\right) / \partial \hat{T}_{2}>0$; this implies that the function $Z\left(\hat{T}_{1}, \hat{T}_{2}\right)$ is increasing in the diffusion delay. Now, an increase on the r.h.s. of (5) requires a decrease in $\hat{T}_{1}$, an increase in $\hat{T}_{2}$ or both. Therefore, the larger the ratio $\Delta_{1} / \Delta_{2}$ is, the longer the diffusion delay is. Similarly, in the second best economy we have:

$$
Z\left(\tilde{T}_{1}, \tilde{T}_{2}\right) \equiv \frac{X^{\prime}\left(\tilde{T}_{1}\right)}{X^{\prime}\left(\tilde{T}_{2}\right)}=\frac{\left[\Delta_{1}-\Delta_{3}+C(1)-C\right]}{\left[\Delta_{2}-\Delta_{4}+C(2)-C(1)\right]},
$$

with $\partial Z\left(\tilde{T}_{1}, \tilde{T}_{2}\right) / \partial \tilde{T}_{1}<0$ and $\partial Z\left(\tilde{T}_{1}, \tilde{T}_{2}\right) / \partial \tilde{T}_{2}>0$. The diffusion delay depends positively on the r.h.s. of (6). 
Using (5) and (6), we get the following results:

Proposition 3 i) The diffusion delay in the decentralized economy (and in the second best solution) is shorter when the degree of product differentiation in duopoly is higher;

ii) The diffusion delay is longer in the second-best solution than in the market economy.

Proof. To show that diffusion is lower in a more differentiated duopoly market, observe that in a decentralized economy diffusion depends on the ratio $\Delta_{1} / \Delta_{2}$. By Property 1 , we know that $\Delta_{1} / \Delta_{2}>1$, implying that $\hat{T}_{2}>\hat{T}_{1}$. Now, with almost independent products, i.e. $\gamma=\delta$, where $\delta>0$ but very small, the ratio $\Delta_{1} / \Delta_{2}$ is close to 1 . Moreover, we have that $\Delta_{1} / \Delta_{2}$ increases in $\gamma$; in fact, $\partial\left(\Delta_{1} / \Delta_{2}\right) / \partial \gamma>0$ for all $\gamma \in(0,1]$. This means that $\Delta_{1} / \Delta_{2}$ assumes the maximum value when products are perfectly substitute, i.e. $\gamma=1$; in such a case, $\Delta_{1} / \Delta_{2}$ tends to be $(\alpha-c+\varepsilon) /(\alpha-c)$ (that is larger than 1). Similar arguments could be used to show that this result holds also in the second-best solution.

Finally, comparing (5) and (6) and after some algebra, we have that $\left[\Delta_{1}-\Delta_{3}+C(1)-C\right] /\left[\Delta_{2}-\Delta_{4}+C(2)-C(1)\right]>\Delta_{1} / \Delta_{2}$ holds for all $\gamma \in$ $(0,1]$. This implies a longer diffusion delay in the second-best solution than in the market economy.

We conclude that higher product differentiation reduces the diffusion delay or, similarly, the time elapsed between the first and second adoption is longer in a duopoly with a higher degree of competition. This result is in accordance with Götz (1999, p. 690) who shows that an increasing degree of competitiveness in monopolistic competition leads to an expansion of the diffusion process.

Moreover, once adopted, the diffusion delay is shorter in the decentralized economy. In effect, since the social planner also takes into account the profit decrease of the first adopter induced by full adoption (which according to Property 2 is larger than the loss in terms of profits induced by the first adoption on the second adopter), he does provide more protection to the first adopter than the market solution. As a consequence, the time elapsed between the two adoptions is lengthened by the social planner compared to the decentralized economy's timing. 


\section{Conclusion}

In this note, we have extend Reinganum's seminal framework (1981a) to account for product differentiation. We show that adding this dimension enriches markedly the discussion around technological diffusion and competition toughness. In particular, we show that the relationship between competition toughness and diffusion is $U$ - shaped, and that the comparison between adoption patterns in the decentralized Vs second-best equilibria tightly depends on the degree of product differentiation. These results are obtained for linear demand functions, and more importantly, in the duopoly case. The oligopoly case is in our agenda. 


\section{References}

[1] Fudenberg and Tirole (1985). "Preemption and Rent Equalization in the Adoption of New Technology", Review of Economic Studies, 52, 383401.

[2] Götz (1999). "Monopolistic Competition and the Diffusion of New Technology", Rand Journal of Economics, 30, 679-693.

[3] Quirmbach (1986). "The diffusion of new technology and the market for an innovation", Rand Journal of Economics, 17, 33-47.

[4] Reinganum (1981a). "On the diffusion of New Technology: A Game Theoretic Approach", Review of Economic Studies, 48, 395-405.

[5] Reinganum (1981b). "Market Structure and the Diffusion of New Technology", Bell Journal of Economics, 12, 618-624.

[6] Singh and Vives (1984). "Price and quantity competition in a differentiated duopoly" Rand Journal of Economics, 15, 546-554. 